\title{
Fractional Fourier transform as a tool for analyzing beam propagation and spherical mirror resonators
}

\author{
Haldun M. Ozaktas \\ Department of Electrical Engineering, Bilkent University, 06533 Bilkent, Ankara, Turkey \\ David Mendlovic \\ Faculty of Engineering, Tel Aviv University, $69978 \mathrm{Tel}$ Aviv, Israel
}

Received April 28, 1994

\begin{abstract}
The complex amplitude distributions on two spherical reference surfaces of given curvature and spacing are simply related by a fractional Fourier transform. The order of the fractional Fourier transform is proportional to the Gouy phase shift between the two surfaces. This result provides new insight into wave propagation and spherical mirror resonators as well as the possibility of exploiting the fractional Fourier transform as a mathematical tool in analyzing such systems.
\end{abstract}

Let $f_{0}(x, y)$ denote the complex amplitude distribution at the plane $z=0$ of light propagating in the positive $z$ direction. We are interested in the amplitude distribution at other planes $z \neq 0$. The simplest and usual approach is to employ harmonic expansion, because plane waves are the eigenfunctions of propagation in free space. ${ }^{1}$ Instead, we will expand $f_{0}(x, y)$ in terms of scaled HermiteGaussian functions, which also constitute a complete orthonormal set. Normalized such that its magnitude squared integrates to unity, the $l$ th-order Hermite-Gaussian function is given by

$$
\psi_{l}(u)=\frac{2^{1 / 4}}{\sqrt{2^{l} l !}} H_{l}(\sqrt{2 \pi} u) \exp \left(-\pi u^{2}\right),
$$

where $H_{l}(\cdot)$ is the $l$ th-order Hermite polynomial. We can expand $f_{0}(x, y)$ in terms of these functions as follows:

$$
\begin{aligned}
f_{0}(x, y) & =\sum_{l=0}^{\infty} \sum_{m=0}^{\infty} A_{l m} \frac{1}{s_{0}} \psi_{l}\left(\frac{x}{s_{0}}\right) \psi_{m}\left(\frac{y}{s_{0}}\right) \\
A_{l m} & =\int_{-\infty}^{\infty} \int_{-\infty}^{\infty} f_{0}(x, y) \frac{1}{s_{0}} \psi_{l}\left(\frac{x}{s_{0}}\right) \psi_{m}\left(\frac{y}{s_{0}}\right) \mathrm{d} x \mathrm{~d} y,
\end{aligned}
$$

because $\int_{-\infty}^{\infty}\left|\psi\left(x / s_{0}\right)\right|^{2} \mathrm{~d} x=s_{0}$ for any arbitrary $s_{0}>0$.

We can interpret the function $s_{0}{ }^{-1} \psi_{l}\left(x / s_{0}\right) \psi_{m}\left(y / s_{0}\right)$ as the amplitude distribution at $z=0$ of a twodimensional Hermite-Gaussian beam of order $(l, m)$ with scale parameter $s_{0}$. Then it becomes an easy matter to write the amplitude distribution $f_{n}(x, y)$ at an arbitrary plane $z=z_{n}$, because we know how each of the Hermite-Gaussian components propagates ${ }^{1}$ :

$$
\begin{aligned}
& f_{n}(x, y)=\sum_{l=0}^{\infty} \sum_{m=0}^{\infty} A_{l m} \frac{1}{s_{n}} \psi_{l}\left(\frac{x}{s_{n}}\right) \psi_{m}\left(\frac{y}{s_{n}}\right) \\
& \quad \times \exp \left[i k z_{n}+\frac{i k\left(x^{2}+y^{2}\right)}{2 R_{n}}-i(l+m+1) \zeta_{n}\right] .
\end{aligned}
$$

In this equation $s_{n} \equiv s\left(z_{n}\right)=\sqrt{\pi} w\left(z_{n}\right)$, where $w\left(z_{n}\right)=$ $w(0)\left[1+\left(z_{n} / \bar{z}\right)^{2}\right]^{1 / 2}$ is the beam radius. Thus $s_{0} \equiv$ $s(0)=\sqrt{\pi} w(0)$, where $w(0)$ is the waist radius. The Rayleigh range $\bar{z}$ is related to $s_{0}$ by the relation $s_{0}{ }^{2}=\lambda \bar{z}$. We also have $k=2 \pi / \lambda$, where $\lambda$ is the wavelength. $R_{n} \equiv R\left(z_{n}\right)=z_{n}\left[1+\left(\bar{z} / z_{n}\right)^{2}\right]$ is the radius of curvature of the wave fronts, and $\zeta_{n} \equiv \zeta\left(z_{n}\right)=$ $\arctan \left(z_{n} / \bar{z}\right)$ is the Gouy phase shift. ${ }^{1}$

Equation (4) can be written in a considerably simpler manner in terms of the fractional Fourier transform. ${ }^{2-8}$ (A brief discussion is given at the end of this Letter.) Let us define the circumflexed functions with normalized arguments such that $\hat{f}_{n}\left(x / s_{n}, y / s_{n}\right)=f_{n}(x, y)$, etc. Furthermore, let $\left(\mathcal{F}^{a_{n}} \hat{f}_{0}\right)(u, v)$ denote the two-dimensional $a_{n}$ th-order fractional Fourier transform of $\hat{f}_{0}(u, v)$. Then the amplitude distribution at the plane $z=z_{n}$ is given by

$$
\begin{aligned}
f_{n}(x, y)= & \frac{s_{0}}{s_{n}}\left(\mathcal{F}^{a_{n}} \hat{f_{0}}\right)\left(\frac{x}{s_{n}}, \frac{y}{s_{n}}\right) \exp \left(i k z_{n}-i \zeta_{n}\right) \\
& \times \exp \left[\frac{i k\left(x^{2}+y^{2}\right)}{2 R_{n}}\right],
\end{aligned}
$$

with

$$
a_{n}=\frac{\zeta_{n}}{\pi / 2}
$$

We discuss the interpretation of this result shortly. But first, let us put it to a simple test. Letting $z \rightarrow \infty$, we see that the resulting intensity pattern is simply the magnitude squared of the first-order (ordinary) Fourier transform of $f_{0}(x, y)$, consistent with what we know of Fraunhofer diffraction.

Let us now consider any two planes $z_{1} \neq 0$ and $z_{2} \neq 0$ such that $z_{1}<z_{2}$ and relate the amplitude distribution in these two planes. Equation (5) holds for both planes. Let us introduce the scaled coordinates $u_{2}=x / s_{2}, v_{2}=y / s_{2}, u_{1}=x / s_{1}$, and $v_{1}=y / s_{1}$ on the spherical surfaces with radii $R_{2}$ and $R_{1}$ (see Fig. 1 ). 


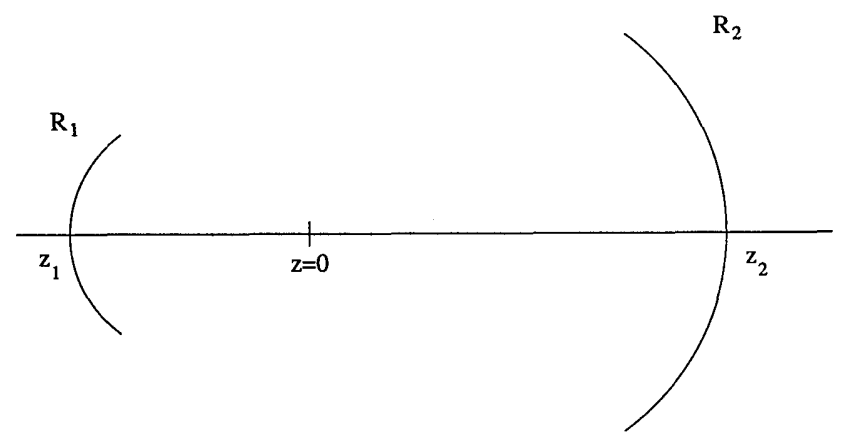

Fig. 1. Spherical surfaces of given radii and separation: the complex amplitude distribution on the second surface is the fractional Fourier transform of that on the first surface. $\hat{g}\left(u_{1}, v_{1}\right)$ and $\hat{h}\left(u_{2}, v_{2}\right)$ denote the complex amplitude distributions on the scaled coordinate systems on surfaces 1 and 2 , respectively. In the figure $z_{1}<0$ and $z_{2}>0$, but the results remain valid if both surfaces are on the same side of the $z=0$ plane.

Working with these spherical reference surfaces enables us to eliminate the final quadratic phase factor in Eq. (5). Then, in terms of our new coordinates, the complex amplitude distribution of light in the two spherical surfaces shown in Fig. 1 can be related in the particularly simple form

$$
\hat{h}\left(u_{2}, v_{2}\right)=\frac{s_{1}}{s_{2}}\left(\mathcal{F}^{a} \hat{g}\right)\left(u_{2}, v_{2}\right),
$$

where

$$
a=a_{2}-a_{1}=\frac{\zeta_{2}-\zeta_{1}}{\pi / 2} .
$$

$\mathcal{F}^{a} \hat{g}$ denotes the ath fractional Fourier transform of $\hat{g}$, and we have dropped the uninteresting phase factor $\exp \left[i k\left(z_{2}-z_{1}\right)-i\left(\zeta_{2}-\zeta_{1}\right)\right]$ from the right-hand side of Eq. (7). The factor $s_{1} / s_{2}$, of course, ensures power conservation. [One derives Eq. (7) from Eq. (5) by first writing $\hat{h}\left(u_{2}, v_{2}\right) \propto$ $\left(s_{0} / s_{2}\right)\left(\mathcal{F}^{a_{n}} \hat{f}_{0}\right)\left(u_{2}, v_{2}\right)$, and a similar equation for $z_{1}$ and then combining them.]

The parameters $a_{1}=2 \zeta_{1} / \pi$ and $a_{2}=2 \zeta_{2} / \pi$ are merely special cases of Eq. (6). At $z=z_{1}$, we have the $a_{1}$ th fractional transform of the distribution at $z=0$, which implies that at $z=0$ we have the $-a_{1}$ th fractional transform of the distribution at $z=z_{1}$. At $z=z_{2}$, we have the $a_{2}$ th fractional transform of the distribution at $z=0$. Thus, at $z=z_{2}$, we have the $\left(a_{2}-a_{1}\right)$ th transform of the distribution at $z_{1}$. This is the essential content of Eq. (7).

It is known that for a proper choice of parameters it is possible to obtain an exact Fourier-transform relation between two spherical surfaces (choose $a=1$ ). What we have shown is that for other values of the parameters we obtain a fractional Fourier-transform relation. Given any two surfaces, as in Fig. 1, all we need to do to find the order $a$ of the fractional Fouriertransform relation existing between these two surfaces is to find the Rayleigh range and waist location of a Gaussian beam that would fit into these surfaces and then calculate $a$ from Eq. (8).

The complex amplitude distribution with respect to any given reference sphere can be mapped harm- lessly onto another reference sphere by using a lens of appropriate focal length. Conversely, the effect of an ideal thin lens can be interpreted merely as a change of the spherical reference surface, with no change in the amplitude. Thus any system of concatenated lenses and segments of free space with any choice of spherical or planar input/output reference surfaces can be analyzed within this framework, as consecutive fractional Fourier transforms. As a simple example, if we wish to obtain an exact fractional Fourier-transform relation between two planar-rather then spherical-surfaces, we can use appropriately chosen and situated lenses to cancel the overall spherical phase factors. (Lohmann's Type I and Type II systems ${ }^{7}$ may be interpreted as special cases. In general, the total amount of spherical phase correction can be distributed in a much more flexible way.)

We may also think of a complex amplitude distribution riding on a Gaussian beam wave front. The spatial dependence of the wave front as the wave propagates is like a carrier defining spherical surfaces, on top of which the complex amplitude distribution rides, being fractional Fourier transformed in the process.

Hermite-Gaussian functions are not strictly eigenfunctions of free space, although they do preserve their profile within a scaling factor. However, they are eigenfunctions of periodic lens systems and spherical mirror resonators. Thus it will be instructive to relate the above results to spherical mirror resonators. Let us now interpret Fig. 1 as a resonator. Assume that the complex amplitude distribution of light at, say, the waist plane is known. After one round trip, we will observe at the same plane the 2ath fractional Fourier transform of the initial distribution, where $a$ is given by Eq. (8). (This is because the mirror precisely reverses the quadratic phase factor so that we get twice the effect on completing a round trip.) That is, one round trip in the resonator is described by a fractional Fourier transform operation. In general, this $2 a$ th fractional Fourier transform is not of the same functional form as the initial distribution. If the initial distribution is to be a mode of the resonator, it must preserve its functional form after a round trip. That is, it must be an eigenfunction of the fractional Fourier-transform operation. But eigenfunctions of the fractional Fourier transform are known to be the Hermite-Gaussian functions ${ }^{8}$ (which are well known to be the modes of spherical mirror resonators).

It is possible for one to express $a$ [as given by Eq. (8)] in terms of the radii of curvature and spacing of the resonator mirrors by first calculating the Rayleigh range and waist location of a Gaussian beam that fits such a resonator and then calculating the Gouy phase shift. In general this results in a complicated expression. For symmetrical resonators with $\left|R_{1}\right|=\left|R_{2}\right| \equiv R$, we obtain

$$
a \frac{\pi}{2}=\arctan \left[\frac{(2 R / d-1)^{1 / 2}}{R / d-1}\right]
$$


The range of the arctangent function in this expression is chosen such that $a \pi / 2$ monotonically decreases from $\pi$ to 0 as $R / d$ increases from $1 / 2$ to $\infty$. For $R / d<1 / 2$, the resonator is unstable.

Let us examine the important special case of the symmetrical confocal resonator in which the radii of the mirrors equal their spacing. In this case, half a round trip through the resonator corresponds to the ordinary Fourier transform, that is, $a=1$. Lipson and Lipson ${ }^{9}$ have also discussed so-called quasi-confocal resonators, in which the beam profile repeats itself not after one round trip but after several round trips. Such systems are easily analyzed within the framework of fractional Fourier transforms. ${ }^{10}$ For instance, if for the resonator under question we have $a=2 / 3$ and thus $2 a=4 / 3$, after three round trips the beam profile will repeat itself.

The well-known stability (or confinement) condition for spherical mirror resonators ${ }^{1}$ can be cast in a particularly simple form in terms of the parameter $a$,

$$
0 \leq[\cos (a \pi / 2)]^{2} \leq 1
$$

Note that, as long as $a$ is real, we have a stable resonator. In this Letter we have implicitly assumed that $a$ and the Rayleigh range $\bar{z}$ are real, which means that we have implicitly assumed stable resonators. Unstable resonators are described by values of $a$ that are not real. Elaboration of this issue must be left for a future discussion.

In conclusion, we have cast the well-known formulation and properties of Hermite-Gaussian beams and spherical mirror resonators in a simple and transparent form in terms of a transform with several interesting and relatively well-studied properties.

Finally, so that the reader may easily verify our results, we give the definition of the ath-order fractional Fourier transform $\left(\mathcal{F}^{a} \hat{f}\right)(u, v)$ of $\hat{f}(u, v)$ :

$$
\begin{aligned}
\left(\mathcal{F}^{a} \hat{f}\right)(u, v)= & \int_{-\infty}^{\infty} \int_{-\infty}^{\infty} B_{a}\left(u, v ; u^{\prime}, v^{\prime}\right) \hat{f}\left(u^{\prime}, v^{\prime}\right) \mathrm{d} u^{\prime} \mathrm{d} v^{\prime} \\
B_{a}\left(u, v ; u^{\prime}, v^{\prime}\right)= & \sum_{l=0}^{\infty} \sum_{m=0}^{\infty} \exp (-i a l \pi / 2) \exp (-i a m \pi / 2) \\
& \times \psi_{l}(u) \psi_{m}(v) \psi_{l}\left(u^{\prime}\right) \psi_{m}\left(v^{\prime}\right)
\end{aligned}
$$

The kernel can also be written in closed form, ${ }^{8}$ but this form is more useful in the present context. The $a=1$ st transform is the ordinary Fourier transform. The ath transform of the $a^{\prime}$ th transform is equal to the $\left(a+a^{\prime}\right)$ th transform. The Hermite-Gaussian functions are eigenfunctions of the fractional Fourier transform operator $\mathcal{F}^{a}$ :

$$
\begin{aligned}
\mathcal{F}^{a}\left[\psi_{l}(u) \psi_{m}(v)\right]= & \exp (-i a l \pi / 2) \exp (-i a m \pi / 2) \\
& \times\left[\psi_{l}(u) \psi_{m}(v)\right]
\end{aligned}
$$

Other properties of this by now fairly well-studied transform may be found in Refs. 2-8.

The benefit of collaborating with A. W. Lohmann of the University of Erlangen-Nürnberg on various phases of our research on fractional Fourier transforms is gratefully acknowledged.

\section{References}

1. B. E. A. Saleh and M. C. Teich, Fundamentals of Photonics (Wiley, New York, 1991).

2. H. M. Ozaktas and D. Mendlovic, Opt. Commun. 101, 163 (1993).

3. D. Mendlovic and H. M. Ozaktas, J. Opt. Soc. Am. A 10, 1875 (1993).

4. H. M. Ozaktas and D. Medlovic, J. Opt. Soc. Am. A 10, 2522 (1993).

5. D. Mendlovic, H. M. Ozaktas, and A. W. Lohmann, Appl. Opt. 33, 6188 (1994).

6. A. C. McBride and F. H. Kerr, IMA J. Appl. Math. 39, 159 (1987).

7. A. W. Lohmann, J. Opt. Soc. Am. A 10, 2181 (1993).

8. H. M. Ozaktas, B. Barshan, D. Mendlovic, and L. Onural, J. Opt. Soc. Am. A 11,547 (1994).

9. S. G. Lipson and H. Lipson, J. Opt. Soc. Am. A 10, 2088 (1993); Optical Physics, 2nd ed. (Cambridge U. Press, Cambridge, 1981), p. 191.

10. D. Mendlovic, H. M. Ozaktas, and A. W. Lohmann, Opt. Commun. 105, 36 (1994). 